\title{
OPTICAL PUMPING OF COPPER METASTABLE ATOMS USED FOR LIFETIME MEASUREMENTS OF HIGH VIBRATIONAL LEVELS OF ELECTRONICALLY EXCITED COPPER HALIDES FORMED BY REACTIVE COLLISIONS
}

\author{
P. KOWALCZYK ${ }^{(1)}$, I. HIKMET and N. SADEGHI \\ Laboratoire de Spectrométrie Physique (URA 08), Université Joseph-Fourier/Grenoble I, \\ BP. 87, F-38402 Saint-Martin d'Hères cedex, France
}

\begin{abstract}
We present a new method for lifetime measurements of excited copper halides formed by reactive collosion of a metastable ${ }_{\mathrm{D}}^{\mathrm{D}}$ copper atom with a halogen compound. The method is applied for excited states in $\mathrm{CuF}, \mathrm{CuCl}$ and $\mathrm{CuBr}$, including high vibrational levels. Additionally, the molecular constants for the $A^{3} \Pi_{1}$ and a new $A^{\prime} \Pi_{2}$ states in CuBr are reported.
\end{abstract}

Spectra of copper halides Cux are fairly complex and not fully understood. The large spin-orbit interaction in $\mathrm{Cu}$ mixes triplet states of the same symmetry, allowing electronically excited states of both multiplicities to radiate to the ground state $x^{1} \Sigma^{+}$. Consequently, assignment of all of the experimentally observed states is not straightforward. This is particularly the case when CuX is formed following the reactive collision of a copper atom with a halogen compound, which populates very high vibrational levels of electronically excited cux molecules and leads to a very dense emission spectrum [1,2]. Radiative lifetime measurement, following excitation from the ground state with a short laser pulse, is a powerful method to discriminate different excited states. In particular, triplet states are expected to have longer lifetimes than the singlets. However, due to the diagonal nature of the Franck-Condon factors in these molecules, the measurements $[3,4]$ were restricted to the lowest vibrational levels in each excited state, which can be optically excited from the thermally populated low $\mathrm{v}$ levels of the ground state.

To overcome this difficulty, we have developed an alternative method of lifetime measurements in copper halides, free from the restriction mentioned above. The method is based on observation of the temporal decay of population of the excited levels, following a fast decrease in the source term populating these levels.

A fast flow reactor, combined with a hollow-cathode sputtering source, was used to carry copper metastable atoms $\mathrm{Cu}^{*}\left({ }^{2} \mathrm{D}\right)$ to the reaction zone [2]. Here the reactant gas (5\% of $\mathrm{F}_{2}$, $\mathrm{Cl}_{2}$ or $\mathrm{Br}_{2}$ diluted in a noble gas) was added, producing a bright chemiluminescence flame. It was due to the reaction of the metastable copper atoms with halogen molecules: 


$$
\begin{aligned}
& \mathrm{Cu}^{*}\left({ }^{2} \mathrm{D}\right)+\mathrm{x}_{2} \longrightarrow \mathrm{Cux}^{*}(\mathrm{n}, \mathrm{v})+\mathrm{X} \\
& \mathrm{Cux}^{*} \longrightarrow \mathrm{CuX}+\mathrm{hw}
\end{aligned}
$$

where $\mathbf{n}$ and $\mathbf{v}$ denote particular electronic state and vibrational level. Under steady-state conditions we observed chemiluminescence from a given molecular state with a constant intensity, corresponding to equilibrium between formation of $\mathrm{Cux}^{*}$ molecules in reaction (1) and radiative (2) and collisional decay. Then a short pulse of a copper vapour laser (pulse duration $\approx 50$ ns, repetition rate $6.5 \mathrm{kHz}$, energy $1 \mathrm{~mJ} /$ pulse) depopulated $\mathrm{Cu}^{*}\left({ }^{2} \mathrm{D}\right)$ metastable atoms in the reaction zone [2]. Since the chemiluminescence of copper halides is almost uniquely due to the chemical reaction (1) involving metastable copper atoms and halogen molecules [2], we were able to observe an exponential decay of population of the excited molecular states. The decay of the (spectrally resolved) chemiluminescence signal was averaged with a transient 64 channel analyzer and sent to the microcomputer for further data treatment.

The typical shape of the time resolved chemiluminescence is shown in Fig.1. The first part of the curve, corresponding to the exponential decay, allows to determine the lifetime of the observed molecular state. Using the experimental method presented above we measured lifetimes of several excited states in CuF, CuCl and CuBr molecules (Fig.2), including high vibrational levels. Results are presented in Table I. A discrepancy of some of our results with the previous measurements [4] will be discussed in the forthcoming paper.

Additionally, we have examined in detail chemiluminescence of CuBr molecule in the spectral range of 465-535nm. Beside of the known $A^{3} \Pi_{1} \longrightarrow x^{1} \Sigma^{+}$system a new band system was observed in this range, corresponding to the transition from a formerly unknown excited $A^{\prime}$ state to the ground $X$ state. The most plausible assignment of the $A^{\prime}$ state is $\Pi_{2}$, i.e. as the $\Omega=2$ fine structure component of the ${ }^{{ }^{n}} \Pi$ manifold, to which belongs also the $A^{3} \Pi_{1}$ state. The molecular constants of the $A^{\circ}$ state were investigated and the vibrational constants of the $A^{3} \Pi_{1}$ state were determined with precision superior to previous measurements (Table II).

\section{REFERENCES}

$/ 1 /$ BALTAYAN, $\mathrm{P}$, HARTMANN, $\mathrm{F}$, PEBAY-PEYROULA, $\mathrm{J}$, SADEGHI, $N$, Chem. Phys. 120 (1988) 123.

/ 2/ BALTAYAN, P, HARTMANN, F, HIKMET, I, PEBAY-PEYROULA, J, SADEGHI, N, Chem. Phys. Lett. 160 (1989) 549.

/3/ DELAVAL, J , LEFEBVRE, $Y$, BOCQUET, $H$, BERNAGE, $P$, NIAY, $P$, Chem. Phys. 111 (1987) 129 .

/4/ LEFEBVRE, Y, DELAVAL, J, BERNAGE, P, NIAY, P, Chem.Phys.Lett. 139 (1987) 212 . 
Table $I$

Measured lifetimes of CuF, CuCl and CuBr states (in us)

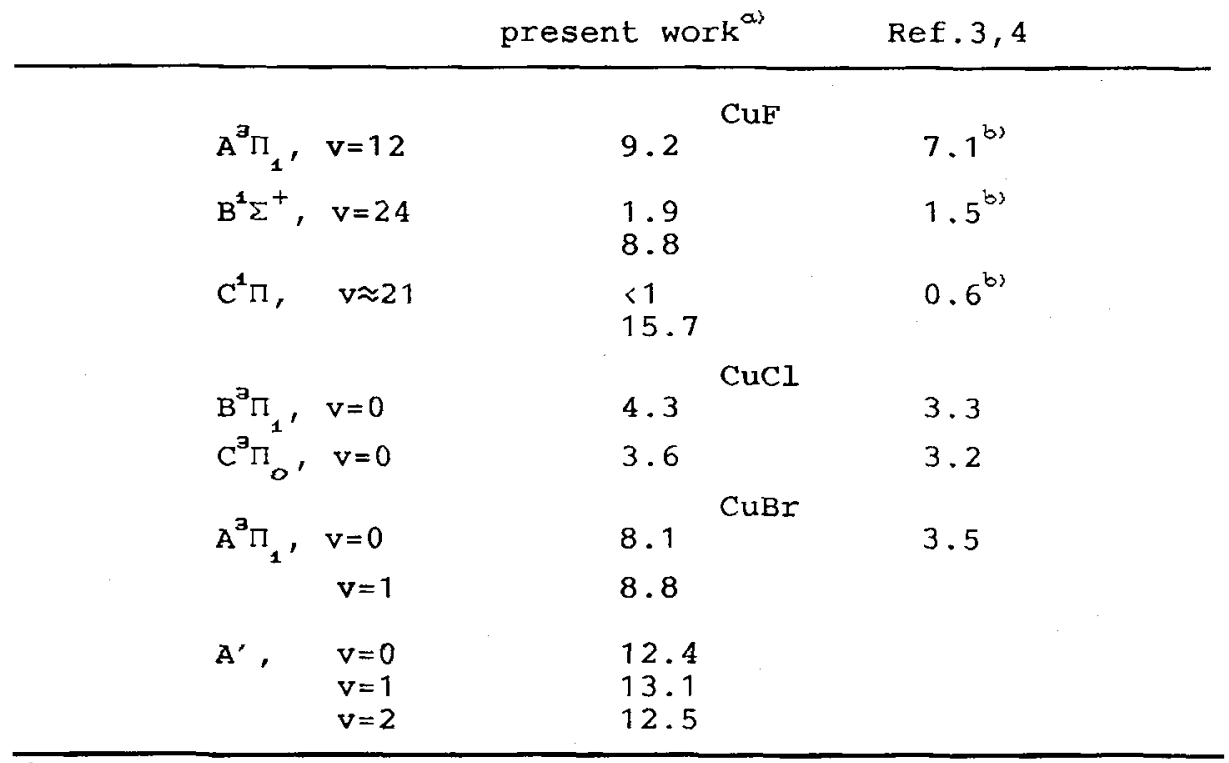

a) experimental error is $\pm 1 \mu \mathrm{s}$

${ }^{b 3}$ measured for $\mathrm{v}=0$

Table II

The molecular constants for the $A^{3} \Pi_{1}$ and $A^{\prime}{ }^{3} \Pi_{2}$ states. All quantities are in $\mathrm{cm}^{-1}$. The error of a constant quoted in parenthesis is one standard deviation.

\begin{tabular}{|c|c|c|}
\hline & $A^{3} \Pi_{1}$ state & $A^{\prime}{ }^{3} \Pi_{2}$ state \\
\hline$T_{\theta}$ & $20496.06(16)$ & $19828.21(16)$ \\
\hline$\omega_{0}$ & $297.69(13)$ & $294.49(12)$ \\
\hline$\omega_{0} x_{0}$ & $1.332(32)$ & $1.525(26)$ \\
\hline$\omega_{0} y_{0}$ & $-0.0125(23)$ & $0.0239(16)$ \\
\hline $\mathrm{B}$ & $0.96509 \cdot 10^{-1}$ as & $0.985(5) \cdot 10^{-1}$ \\
\hline$\alpha$ & $0.65 \cdot 10^{-3}$ & $0.65 \cdot 10^{-3}$ as \\
\hline D。 & $0.389 \cdot 10^{-7} a$ & $0.389 \cdot 10^{-7}$ as \\
\hline
\end{tabular}

a) Taken from G.Mishra et al, J.Mol.Spectrosc. 85 (1981), 245. 


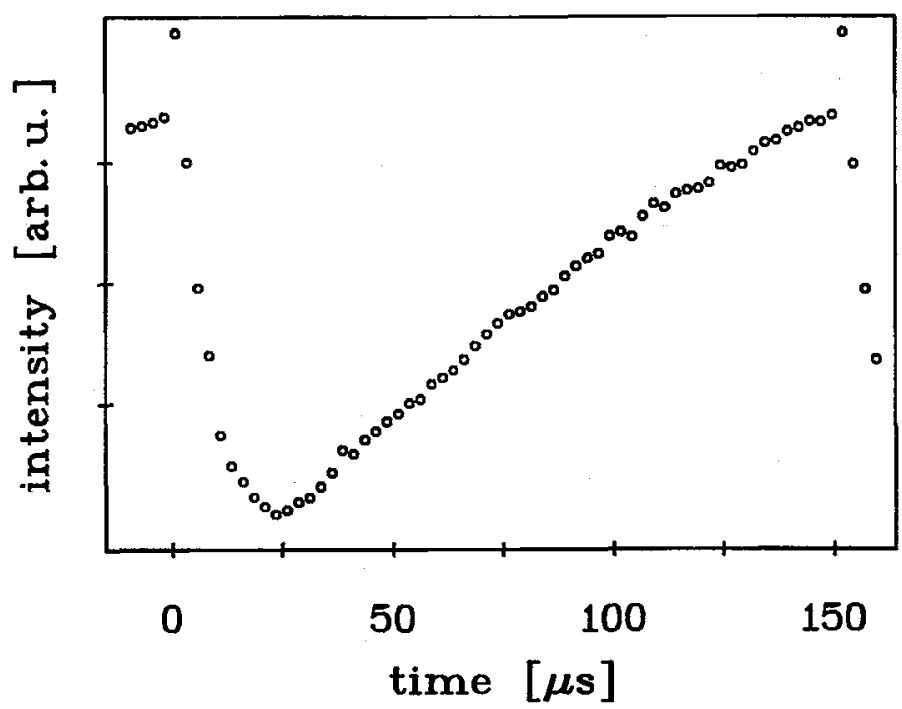

Fig.1. Time evolution of the chemiluminescence signal for $B^{3} \Pi_{1} \longrightarrow$ $\mathrm{x}^{1} \Sigma^{+}(0,0)$ band in CuCl at $488.1 \mathrm{~nm}$. The sharp peaks at $t=0$ and $t=150 \mathrm{ks}$ correspond to a scattered light from the two consecutive laser pulses.

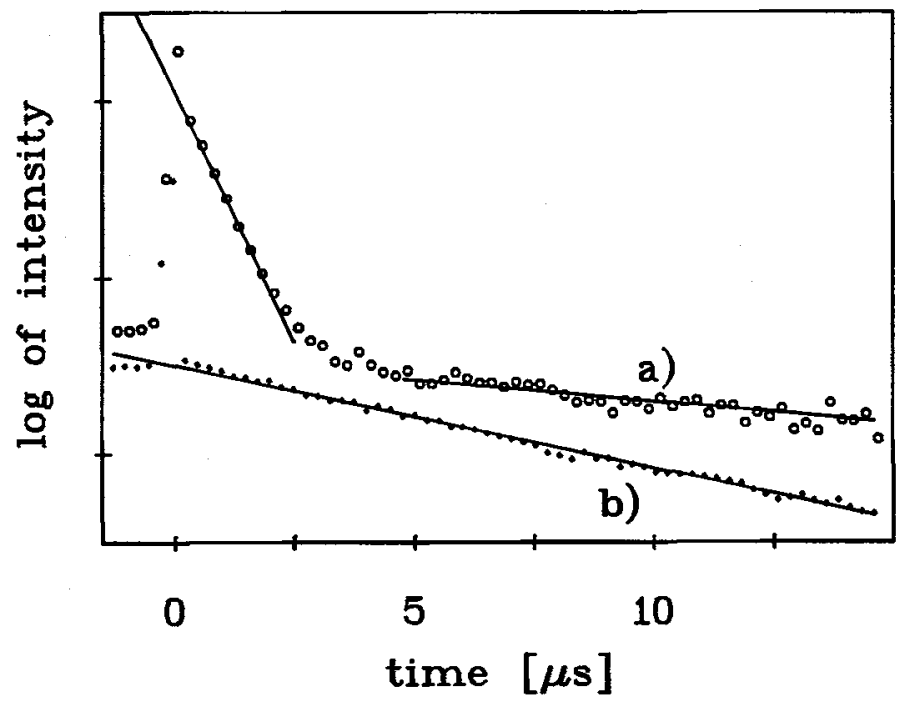

Fig.2. Logaritmic plot of the time resolved chemiluminescence signal for a) $\mathrm{C}^{1} \Pi \longrightarrow \mathrm{x}^{1} \Sigma^{+}$transition in CuF at $485.8 \mathrm{~nm}$, at the head-of-heads of $\Delta \mathrm{v}=0$ system, formed around $\mathrm{v}=21$ (open circles); b) $\mathrm{A}^{3} \Pi_{1} \longrightarrow \mathrm{x}^{1} \Sigma^{+}(0,0)$ band in $\mathrm{CuBr}$ at $487.9 \mathrm{~nm}$ (dots). The peaks at $t=0$ correspond to a scattered laser light. The straight lines represent least squares fits to the experimental points. 